\title{
Cerebellar cavernous malformation in pediatric patients: defining clinical, neuroimaging, and therapeutic characteristics
}

\author{
Friederike Knerlich-Lukoschus, MD, PhD, ${ }^{1,2}$ Paul Steinbok, MBBS, FRCSC, ${ }^{1}$ \\ Christopher Dunham, MD, FRCPC, ${ }^{3}$ and David Douglas Cochrane, MD, FRCSC ${ }^{1}$
}

1Division of Pediatric Neurosurgery, British Columbia Children's Hospital and Department of Surgery, University of British Columbia; ' Division of Anatomical Pathology, Department of Pathology and Laboratory Medicine, British Columbia Children's Hospital, University of British Columbia, Vancouver, British Columbia, Canada; and 'Department of Neurosurgery, University Hospital of Schleswig-Holstein Campus Kiel, Germany

\begin{abstract}
OBJECT Cerebellar cavernous malformations (CCMs) have not been specifically described in the pediatric age group. Authors of this study, after considering the published literature, describe the characteristic clinical, radiological, and surgical features of CCM in children.

METHODS Patients younger than 18 years of age who were known to have CCM and had undergone surgery between 1992 and 2014 at the authors' institution were reviewed. Pediatric CCM cases reported in the literature (case reports and cases included in series on CMs in the pediatric age group) were also analyzed for specific features of this entity.

RESULTS Four male patients and 1 female patient (2.5-14 years of age) with CCM presented acutely with severe headache followed by cerebellar dysfunction. In all patients, neuroimaging (cranial CT and MRI) demonstrated hemorrhagic cerebellar lesions with heterogeneous T1 and T2 signal intensities and hyperintense blooming on susceptibilityweighted imaging. The lesions reached large sizes exhibiting spherical, cystic, and often "pseudotumoral" morphology. In 3 patients, developmental venous anomalies (DVAs) were found. In 4 of the 5 patients, the CCMs and hematomas were totally removed. All patients had a clinically excellent functional outcome without surgical complication and with complete resolution of their presenting symptoms.
\end{abstract}

CONCLUSIONS Cerebellar CMs occur in all pediatric age groups and display characteristic clinical and imaging features. In children, CCMs reach large sizes and can result in massive hemorrhage, often leading to a possible diagnosis of hemorrhage into a tumor. An associated DVA is quite common. Surgery is a safe and efficient treatment option with excellent outcomes in patients.

http://thejns.org/doi/abs/10.3171/2015.1.PEDS14366

KEY WORDS cavernous malformation; cavernoma; cerebellum; pediatric age group; surgical treatment; neuroimaging; outcome; vascular disorders

$\mathrm{C}$ AVERNOUs malformations (CMs), also known as "cavernous angiomas," "cavernous hemangiomas," ties in the brain, with an estimated prevalence of $0.4 \%$ to $0.8 \%$ in the general population. ${ }^{4,12,21,28,33,34}$ In the pediatric age group, their prevalence is not known because of small numbers of patients in age-mixed reports. ${ }^{4}$ In pediatric series, CMs represent $1.7 \%-18 \%$ of all vascular growths. ${ }^{23,25}$

In children, these low-flow vascular malformations are one of the main causes of cerebral hemorrhage besides arteriovenous malformations. . $^{13,24,27,29,35}$ Cavernous malformations occurring in children differ from those in adults as they commonly have atypical neuroimaging at diagnosis, show more rapid rates of growth, and have larger dimensions and a greater likelihood of hemorrhage at presentation. , $14,26,27$ Cavernous malformations are located throughout the CNS in the pediatric age group. ${ }^{24,27}$ Most

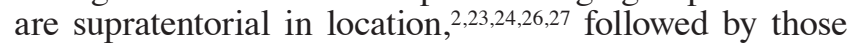

ABBREVIATIONS BCCH = British Columbia Children's Hospital; $\mathrm{CCM}=$ cerebellar cavernous malformation; $\mathrm{CM}=$ cavernous malformation; $\mathrm{CN}=\mathrm{cranial}$ nerve; $\mathrm{CTA}=\mathrm{CT}$ angiography; DVA = developmental venous anomaly; MRA = MR angiography; SWI = susceptibility-weighted imaging.

SUBMITTED July 21, 2014. ACCEPTED January 16, 2015.

INCLUDE WHEN CITING Published online May 22, 2015; DOI: 10.3171/2015.1.PEDS14366.

DISCLOSURE The authors report no conflict of interest concerning the materials or methods used in this study or the findings specified in this paper. 
in the brainstem and less frequently in the cerebellum..$^{10,31}$ de Oliveira et $a .^{10}$ reported that CCMs in adults exhibit specific clinical characteristics. Except for a few case reports, however, CCMs in pediatric patients have not been described. ${ }^{11,16,19}$ In the present study, we describe the clinical and radiological presentation and surgical treatment of CCMs in patients presenting at an age less than 18 years.

\section{Methods}

Ethics approval for this study was obtained from the Clinical Research Ethics Board of British Columbia Children's Hospital (BCCH).

\section{Patient Population and Clinical Data}

The prospectively maintained pediatric neurosurgery electronic database of $\mathrm{BCCH}$ was queried for patients younger than 18 years of age who had been treated for cranial CMs from 1992, when MRI became available at $\mathrm{BCCH}$, to 2014. For the purposes of our study, we then selected patients who had undergone surgery for CCMs and had a histologically confirmed diagnosis. Their charts were retrospectively reviewed for clinical presentation, imaging and surgical features, histopathology, and outcome. All patients had undergone cranial CT and MRI before surgery and MRI after surgery.

\section{Literature Review}

PubMed was searched for the following terms: "cavernoma" or "cavernous angioma" and "pediatric" or "children" and "cerebellar" or "cerebellum." Only articles in English (and 1 in French) were considered. We excluded studies in which pediatric and adult age ranges were mixed and in which cerebellar location was mentioned without providing any patient details, clinical symptoms, or imaging features. ${ }^{4,6}$

\section{Results}

From 1992 to 2014, 27 patients under the age of 18 years were managed by the neurosurgical service at $\mathrm{BCCH}$ for intracranial CMs; 23 were surgically treated and 4 were managed conservatively. Five of these patients had CCMs and were surgically treated. Diagnosis was pathologically confirmed in all 5 patients.

\section{Patient Age, Sex, and Clinical Presentation}

There were 4 male patients and 1 female patient whose ages ranged from 2.5 to 14 years, with a mean age of 7 \pm 4.6 years at presentation (Table 1). All patients were asymptomatic prior to presenting with acute or rapidly progressing headaches.

\section{Case 1}

This 11-year-old boy presented with a 2-week history of the sudden onset of occipital headaches and vomiting. He had intermittent fever and was treated for gastritis in an outpatient clinic. When the symptoms did not resolve and he developed gait ataxia and double vision, he was transferred to BCCH. On arrival, he was cachectic as a result of vomiting, was complaining of severe occipital headaches, and had a left sixth cranial nerve $(\mathrm{CN})$ palsy, ataxia, and left-sided dysmetria.

\section{Case 2}

This 5-year-old boy presented with a 14-day history of increasing headaches. During transport in our hospital, he experienced one syncopal episode. He did not have papilledema, dysmetria, or ataxia. His $\mathrm{CN}$ examination was normal.

\section{Case 3}

This 14-year-old girl had a 4-day history of occipital headaches. Her neurological examination was normal.

\section{Case 4}

This 3-year-old boy was admitted for increasing headaches and unsteadiness of gait, which started a few days before admission. He was neurologically intact except for gait ataxia. There was a history of familial CM, as his father was known to have multiple intracranial CMs.

\section{Case 5}

This 2.5-year-old girl presented with the acute onset of severe headaches and a normal neurological examination.

\section{Neuroimaging Lesion Characteristics}

Because of acute presentations, all patients underwent initial cranial CT, which demonstrated mixed-intensity, round or spherical hemorrhagic cerebellar lesions (Figs. 1A, 2A, 3A, 4A, and 5A). In Case 2, calcification was seen on the inferior portions of the lesion (Fig. 2B). Hemorrhage into a tumor was the commonest referring diagnosis.

In all cases, $\mathrm{CT}$ findings prompted MRI sequences, including T1-weighted, T2-weighted, 3D T1-weighted post-Gd injection, susceptibility-weighted, and diffusionweighted (Table 1). In Cases 1, 2, and 4, MR angiography (MRA) was also performed with the time-of-flight technique. Preoperative MRI exhibited spheroid, cystic mass lesions with considerable variations in size. All images showed compression of adjacent crucial anatomical structures, especially in Case 1 (Fig. 1B-F). All lesions exhibited heterogeneous $\mathrm{T} 1$ and $\mathrm{T} 2$ signal intensities and characteristic hypointense blooming on susceptibility-weighted imaging (SWI) sequences with surrounding edema (Figs. $1 \mathrm{D}, 2 \mathrm{E}$ and $\mathrm{F}, 3 \mathrm{C}, 4 \mathrm{D}$ and $\mathrm{E}$, and $5 \mathrm{~B}$ ). Case 3 differed morphologically, with a compact area (Fig. 3C and E) and diffuse multifocal small lesions scattered through adjacent cerebellar cortex (Fig. 3D) creating a more diffuse blooming pattern on SWI (Fig. 3F).

With the benefit of MRI, cavernous malformation was considered the most likely preoperative diagnosis, followed by hemorrhage into a tumor. The presence of developmental venous anomalies (DVAs; Cases 1, 2, and 5; Figs. 1F, 2H, and 5E and F), multifocal supra- and infratentorial lesions, and/or a family history of CM (Case 4; Fig. 4F) supported this diagnosis.

Formal vascular imaging was performed preoperatively (CT angiography [CTA] in Case 3, and MRA in Cases 1, 2, and 4). These studies excluded abnormal arterial feeders but did not reveal the DVA in Cases 1 and 2 . 


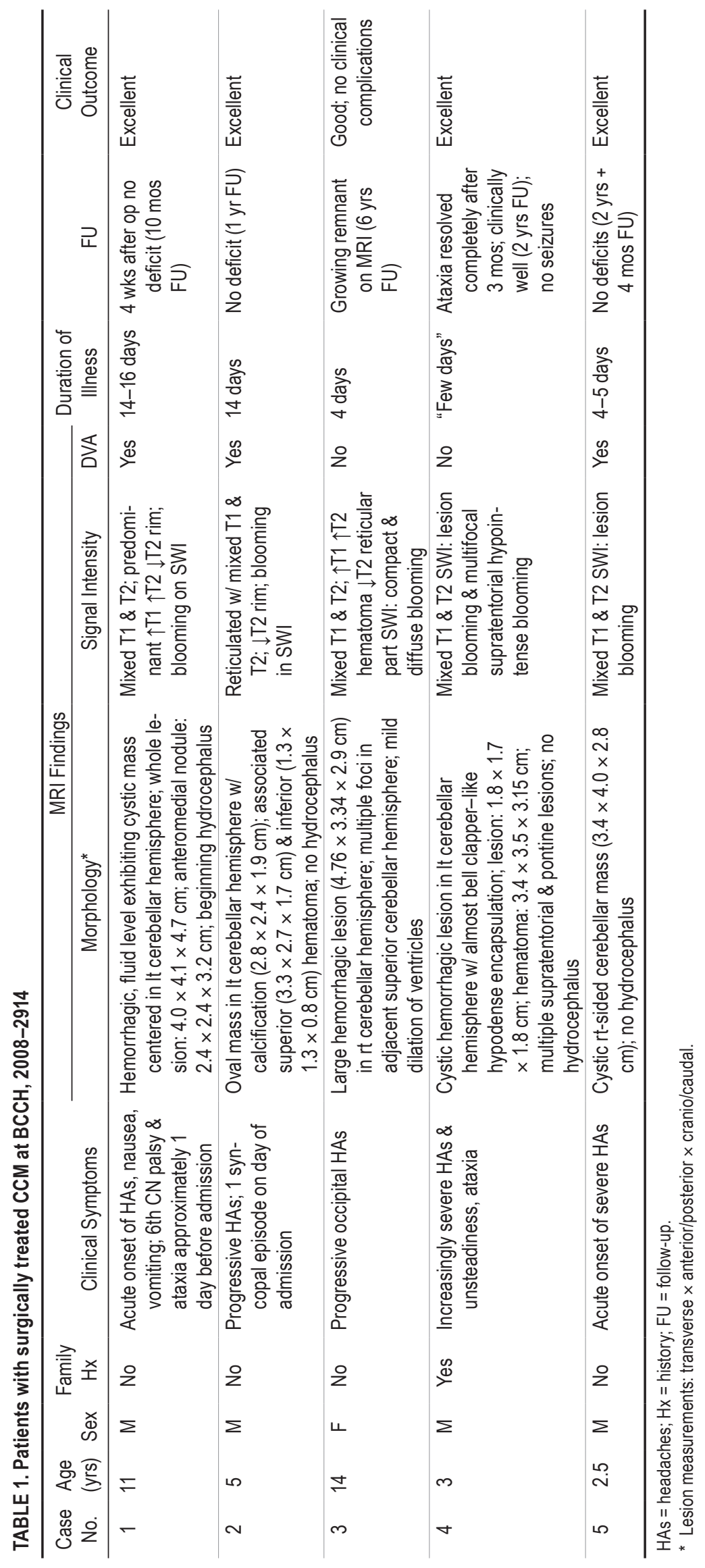



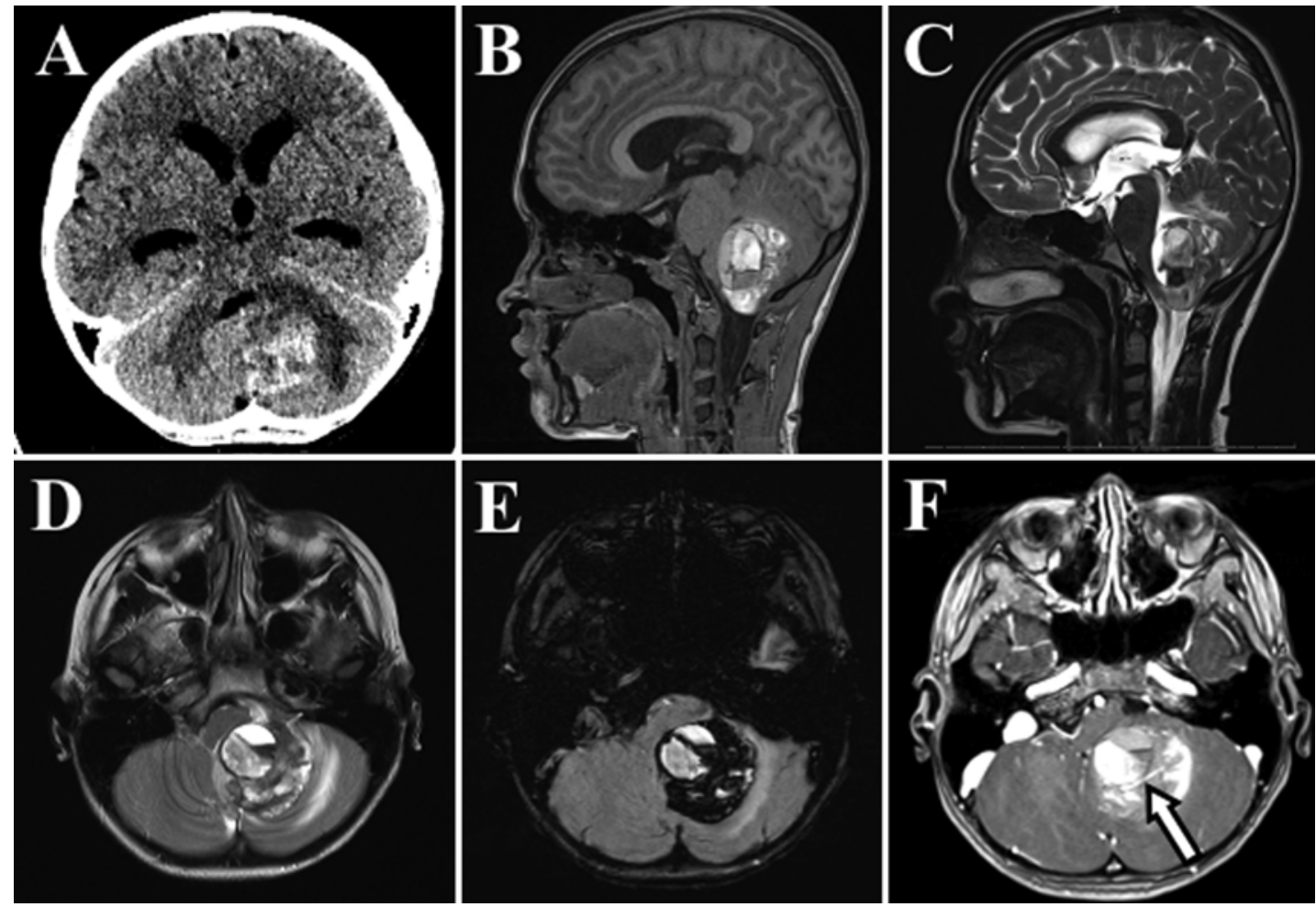

FIG. 1. Case 1. A: Coronal CT scan exhibiting a hemorrhagic lesion centered within the left cerebellar hemisphere and vermis with surrounding edema. There was beginning hydrocephalus with mildly enlarged ventricles and transependymal extravasation. B: Sagittal T1-weighted MRI sequence showing a cystic lesion with heterogeneous, predominantly high T1 signal extending into the left cerebellar tonsil, which appeared caudally displaced. C and D: Sagittal and axial T2-weighted MRI sequences showing the lesion with heterogeneous signal with surrounding low T2 signal rim. There was a well-defined large nodule located anteromedially $(2.4 \times 2.4 \times 3.2 \mathrm{~cm})$ containing a fluid level of different densities. Furthermore, there was edema of adjacent cerebellar parenchyma including the middle cerebellar peduncle and dorsal pontine tegmentum. The mass resulted in distortion of the cerebellar peduncles and medulla at the foramen magnum level. E: Susceptibility-weighted image with signal drop in the lesion lateral to the anteromedial cyst. F: Post-Gd-enhanced sequence did not reveal abnormal enhancing tissue. One vertically oriented vessel exhibited features suspicious for an associated developmental venous anomaly (DVA; arrow), draining into the left inferior petrosal sinus.

All patients underwent whole spinal cord MRI before (Cases 1, 2, 4, and 5) or after (Case 3) surgery. No additional CMs were found.

\section{Surgical Findings}

All patients underwent suboccipital craniotomy and excision of the $\mathrm{CM}$ and its associated hematoma. In Cases 2,3 , and 5 , the lesions seen at surgery exhibited typical berry-like appearances; in Case 4, the lesion was firm and calcified. In Case 2 a venous structure medial and anterior to the lesion, corresponding to MRI findings, was recognized and kept intact. In Case 1 the potential venous structure was not identifiable during resection and appeared to be removed on postoperative MRI. As confirmed with postsurgery MRI, complete removal of the lesion was achieved in all but 1 patient (Case 3). Although the lesion and hematoma in this patient were resected with clean resection margins, the superiorly located diffuse components remained.

\section{Histopathological Lesion Features}

In all cases, the suspected diagnosis of CM was confirmed pathologically. The microscopic specimens showed numerous abnormal blood vessels ranging in size from small to large and were often closely packed together. Focal calcification was found in Cases 2, 4, and 5, and occasional thrombosed vessels were found in Cases 2,3, and 5. No arterial components were seen with Movat's pentachrome stain (Fig. 6). The cerebellar parenchyma, when included in the resected tissue, exhibited acute to subacute remote damage, including acute neuronal necrosis (Case 5), gliosis (Cases 3 and 4), and atrophy. All lesions were associated with an inflammatory reaction, most prominent between the vessels and in the gliotic brain tissue. There were significant numbers of CD68-positive macrophages and occasional perivascular lymphocytes (Cases 1-3).

\section{Surgical Outcome}

In all patients, initial symptoms and clinical manifestations resolved shortly after lesion and clot removal. In Cases 3 and 4, clinically silent imaging changes were noted during follow-up. In Case 3, residual CCM was visualized on postsurgical MRI (Fig. 3). This residual lesion evolved over time with increasing T2 hypointensity (Fig. 3K and L) and hemosiderin blooming on SWI sequences. In addition, a new area of $\mathrm{T} 1$ hyperintensity (1 $\mathrm{cm}$ in diameter) in 

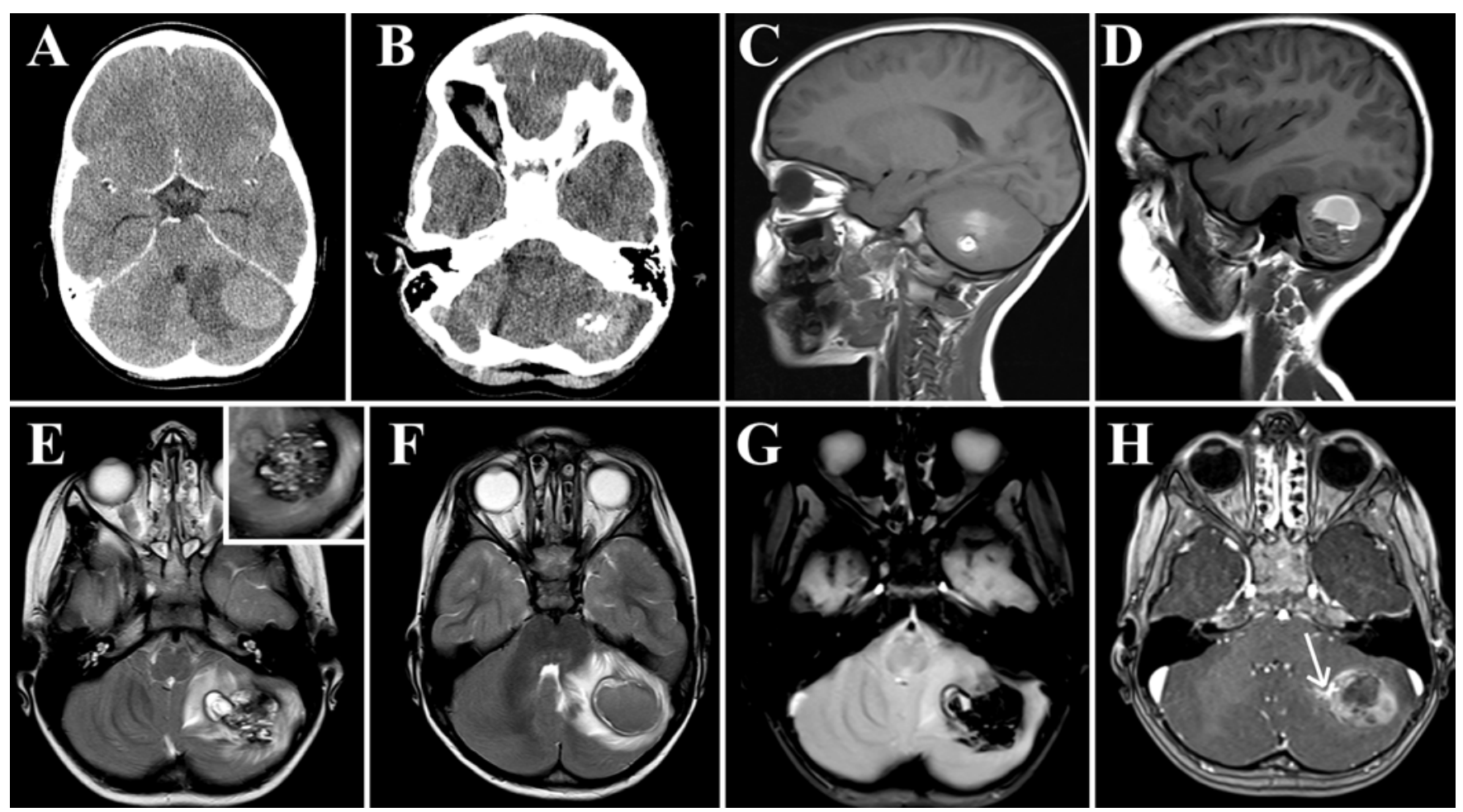

FIG. 2. Case 2. Coronal CT scans obtained on admission, exhibiting a hyperintense, roundish hemorrhaging lesion with surrounding edema (A) and inferior calcifications (B) located in the left cerebellar hemisphere. There was no associated hydrocephalus. Sagittal T1-weighted MRI sequences demonstrating a mixed-density lesion with a hyperintense nodule (C) and superior cystic component (D). Axial T2-weighted MRI sequence (E) showing the reticulated lesion (inset picks up more basal proportions) with mixed, predominately low T2 density, surrounded by a hyperintense edematous rim and associated anteromedial high T2 signal nodule. Another large cystic component was located superior to the lesion with isointense T2 signal intensity (F). On an SWl sequence (G), the lesion was associated with marked blooming artifacts. With contrast enhancement, a vascular structure exhibiting typical features of an associated DVA (arrow) became apparent medially and anteriorly to the lesion $(\mathrm{H})$, presumably draining into a venous sinus in the region of the left tentorium.

the right cerebellar hemisphere (Fig. 3H) appeared following surgery. The patient remained asymptomatic and her scan was unchanged at the 4-year follow-up. The patient in Case 4, who had had numerous bilateral supratentorial and pontine foci of SWI blooming on initial MRI, showed interval enlargement of several of these supratentorial foci and development of one single new lesion within the right temporal lobe. These lesions remained clinically silent at the last follow-up.

\section{Literature Review}

We found 3 case reports and 15 case series providing relevant information on CCM in the pediatric age group. ${ }^{2,5}$, $7-11,13,14,16,19,20,23,24,27,32,35,36,39$ These cases are summarized in Table 2.

\section{Discussion}

\section{Incidence and Sex Distribution of CCM in Children}

The occurrence of CCM in children has been reported to range from $3 \%$ to $13.5 \%$ (mean $8.7 \%$ ) among all cranial CMs. ${ }^{13,20,24,27,40}$ In our experience, CCM accounted for $18 \%$ of CMs. In our surgical series, the occurrence was $21.7 \%$ of cranial CMs.

Cerebellar CMs occur in patients of all ages, with children 11 years and older appearing to be more frequently affected. This contrasts with the bimodal age distribution of CMs in other sites, which shows an infant/early childhood peak at 3 years of age and younger and an older child/teenager peak between 13 and 16 years. ${ }^{17}$

\section{Clinical Presentation}

All CCM cases in our series and those cases reported in the literature presented with clinical signs related to acute cerebellar hemorrhage in previously asymptomatic patients. Headaches often localized in the occipital region had an acute onset and progressive course. Other clinical findings were related to cerebellar dysfunction. One patient in our series had sixth CN palsy. Epstein et al. described a case presenting with ear and facial pain. ${ }^{16}$ Two CCM cases presented with "coma,", ${ }^{11}$ and we are aware of 1 case presenting with a lethal hemorrhage (C. Dunham, personal communication, 2014).

Cerebellar CMs differ from supratentorial CMs that present most often with seizures ${ }^{2,5,23,24,27,39}$ and from brainstem CMs, which become obvious with focal neurological deficits. ${ }^{31,39}$

The time from symptom onset to treatment in our series was 3-16 days. Headaches and vomiting were often treated symptomatically until the patient developed cerebellar dysfunction or CN palsy, which prompted neuroimaging. Patients exhibiting short clinical courses have also 

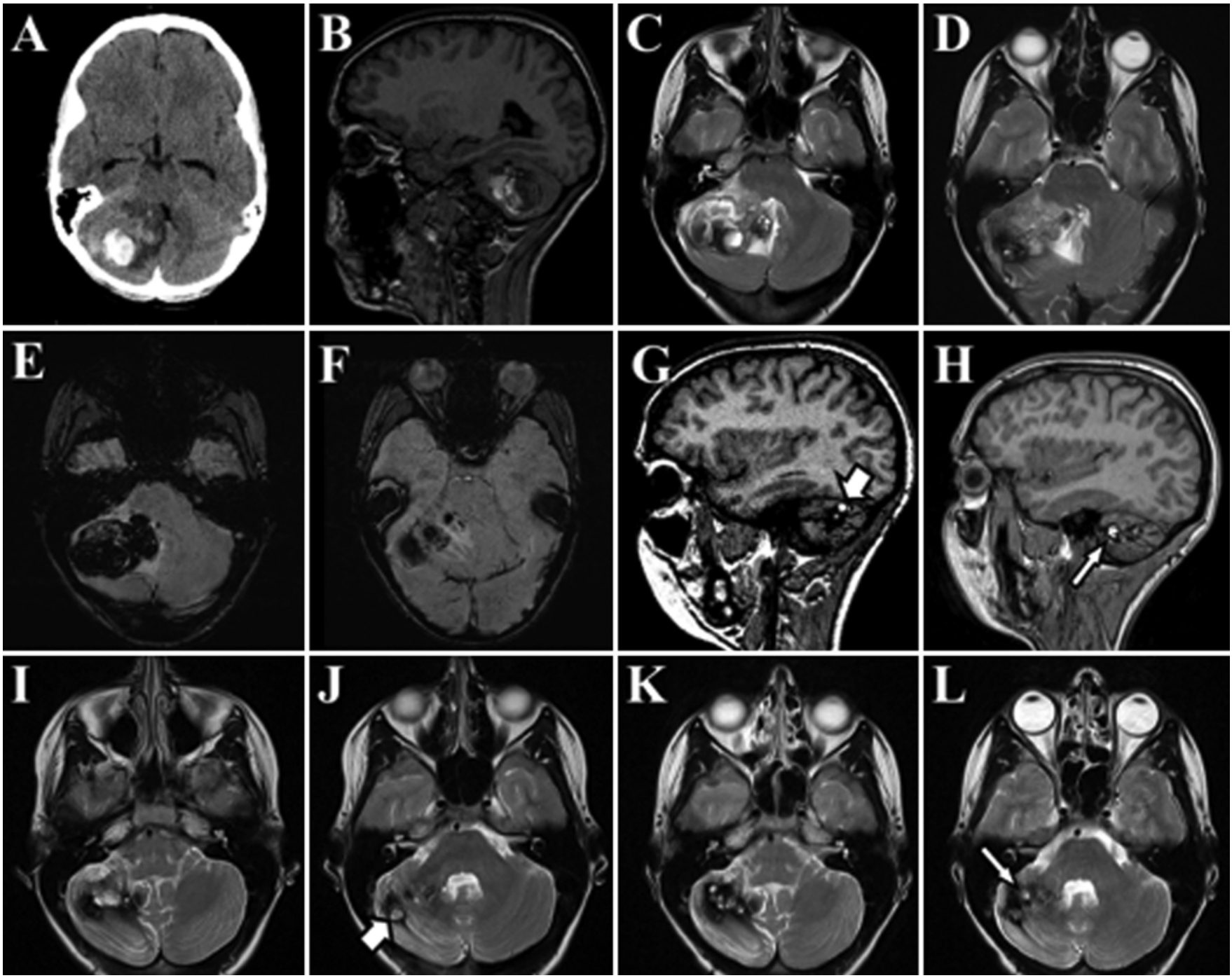

FIG. 3. Case 3. Coronal CT (A) demonstrating a right cerebellar hemisphere hemorrhagic lesion of mixed signal intensity with a hyperintense roundish component and surrounding edema. Sagittal T1-weighted MRI sequence (B) with mixed, partially hyperintense signal lesion. Axial T2-weighted MRI sequence $(\mathbf{C})$ showing heterogeneous signal intensities of cystic and reticulated lesion components with surrounding edematous changes. Superiorly, the lesion proportions appeared to be more diffusely scattered throughout the adjacent parenchyma (D). Susceptibility-weighted images ( $E$ and F) picking up the cystic lesion area corresponding to that in panel $\mathrm{C}$ and a more diffuse blooming pattern scattered through the adjacent superior right cerebellar hemisphere (corresponding to that in panel D). Sagittal T1-weighted images ( $G$ and H) obtained directly after surgery, showing residual lesion (arrows) of mixed T1 intensity with one hyperintense nodule located under the tentorium. Postsurgical axial T2-weighted MRI sequences (I and J) demonstrating multiple foci of residual mixed T2 signal exhibiting lesion components extending from below the tentorium to its inferior margin. Arrow in panel $\mathrm{J}$ depicts residual lesion corresponding to its T1-weighted appearance in panels $\mathrm{G}$, (K), and L. These foci showed dynamic changes over time with an overall increase of T2 hypointensity of hemosiderin deposition suggestive of interval hemorrhage. Moreover, the lesion changed its T2 signaling behavior over time as the posterior infratentorial nodule ( $\mathrm{J}$, arrow) disappeared over time, while 2 years later a new hyperintense foci became apparent more anteriorly (L, arrow).

been reported by Acciarri et al. ${ }^{2}$ In contrast, the patient described by Epstein et al. was first treated conservatively but underwent an operation after a second hemorrhage of the CCM after 4 years. ${ }^{16}$

\section{Imaging Features of Pediatric CCM}

Computed tomography scanning shows cerebellar hemorrhage with significant mass effect. ${ }^{11,16}$ On MRI, these lesions exhibited a spherical or cystic appearance with mixed signal on T1- and T2-weighted sequences, characteristic hypointense T2 lesion rim, and blooming on SWI sequenc- es. This appearance was in keeping with several episodes of bleeding. These hemorrhagic lesions reached significant sizes (Table 1) with distortion of the brainstem or other neighboring structures and were accompanied by perilesional edema..$^{27,37}$ We did not routinely perform CTA preoperatively. Three patients had preoperative MRA. These studies did not add material information to our clinical decision making. The DVAs, when present, were detected on postcontrast T1-weighted sequences in our patients.

Our imaging findings were consistent with those described by Mottolese et al. ${ }^{27}$ In their study, the most com- 

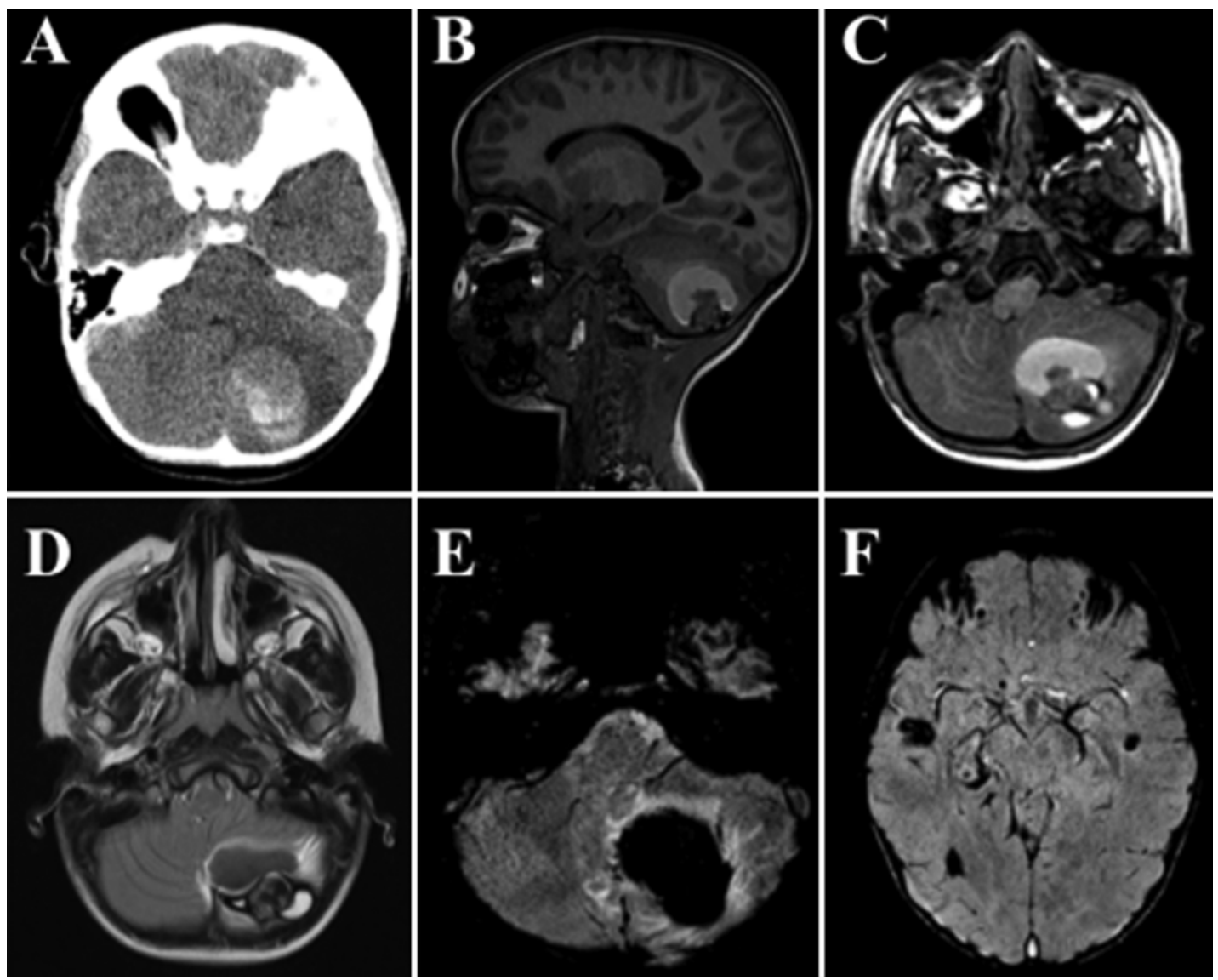

FIG. 4. Case 4. A coronal CT (A) showing a large, almost isointense, partially hyperintense spherical hemorrhagic lesion in the left cerebellar hemisphere. Sagittal (B) and axial (C) T1-weighted MR images showing the different cystic lesion components with mixed signal intensity in keeping with hemorrhages of different ages and acute peripheral bleeding. The lesion resulted in mass effect with compression of the fourth ventricle. Axial T2-weighted MRI sequence (D) demonstrating cystic lesion portions with mixed signal intensities and one exhibiting an almost bell clapper-like hypodense encapsulation. Corresponding SWI sequence (E) with blooming of the lesion. Representative MR image (F) showing multiple supratentorial lesions with characteristic blooming dispersed throughout both cerebral hemispheres due to underlying familial CM.

mon MRI presentation in symptomatic patients was a large, spherical, well-delineated acute or subacute hematoma with sharp margins that contained or were associated with structures of different density signal.

The main differential diagnosis was hemorrhage into a preexisting cerebellar tumor. Because cerebellar tumors are much more common in children than CCMs, the diagnosis of tumor is often the first consideration. The spherical and "cystic" appearance may mimic that of other cystic tumors, for example, pilocytic astrocytomas. ${ }^{25}$ However, features such as an associated DVA, multilocular lesions on SWI, and several hemorrhage episodes (bleeding of different ages) favor $\mathrm{CM}$ as the preoperative diagnosis.

\section{Cerebellar CM-Associated DVA}

The co-occurrence of DVA and CM is recognized in the literature. ${ }^{30,40}$ In the pediatric age group, however, the CM and DVA association has been reported less often. ${ }^{27}$ This contrasts with our observations.

It has been suggested that CMs associated with a DVA have a high propensity to hemorrhage. ${ }^{30}$ Wurm et al. reported that $93.3 \%$ of those harboring an associated DVA had symptomatic hemorrhage..$^{38}$ Abdulrauf et al. compared
CMs with and without an associated venous anomaly and showed that CMs associated with DVAs occur predominantly in the posterior fossa (with a slightly higher proportion of cerebellar $[\mathrm{n}=6]$ compared with brainstem [n $=4] \mathrm{CMs}$ ) with an associated significant higher bleeding and rebleeding risk compared with CMs without associated DVAs. ${ }^{1}$ As such, CCMs in the pediatric age group may be more prone to bleeding than CMs elsewhere in the nervous system.

The clinical importance of a DVA remains unclear. Some authors advocate leaving the DVA intact to prevent hemorrhagic infarction. ${ }^{30}$ In contrast, if the DVA is a promoting factor in the development of cerebral CM, resection of associated DVA components may prevent regrowth of a partially excised CM. ${ }^{15}$ In Case 1 the DVA was removed during $\mathrm{CM}$ extirpation without clinical sequelae.

\section{Histology of Cerebellar CMs}

Our cases exhibited typical pathological characteristics, in accordance with those in the published literature on CMs in cerebellar and other locations..$^{11,27}$ The lesions displayed abnormally dilated vascular spaces of various sizes and degenerative changes with irregular fibrous hyaline 

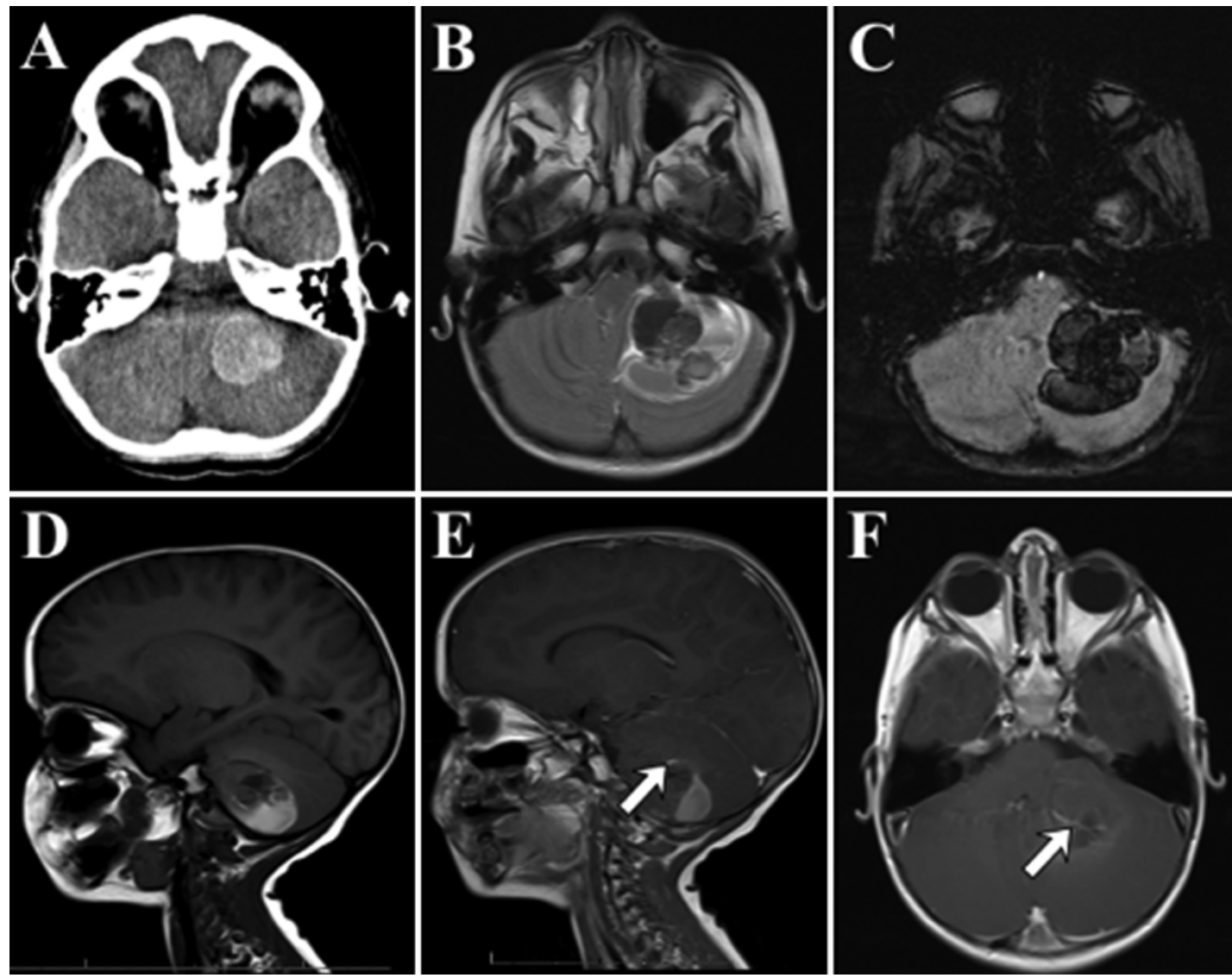

FIG. 5. Case 5. A: Coronal CT obtained on admission, showing a large left cerebellar roundish hemorrhagic lesion with mixed but mostly hyperintense density. B: Axial T2-weighted MR image with mixed density and perilesional edema. There was mass effect on the fourth ventricle and brainstem. C: Corresponding SWI sequence. D: Sagittal T1-weighted MRI sequence with hypo- and hyperintense signal intensities. E and F: Sagittal and axial post-Gd MR images with visible potential DVA but no further pathological enhancement.

thickening, old hemorrhages, and thrombosis. The vessels were packed closely together typically with no normal neural tissue in between. The cerebellar cortex close to the vascular malformation exhibited signs of atrophy and gliosis, also a common finding in the literature on CCM. Additionally, in all of our cases, there was an accompanying inflammatory reaction.

\section{Treatment Options and Outcome}

The overall annual risk of hemorrhage of incidentally diagnosed CMs was reported to be low at $0.4 \%-2 \%$ per year; however, the risk of a second hemorrhage in the next year was estimated to be $4 \%-5 \%$.,22,33 Cavernous malformations in the pediatric age group were reported to be more prone to bleeding with a more dramatic clinical course than those in adults. ${ }^{18,20,26}$ Surgical removal of symptomatic CCM is considered the treatment of choice with a good overall outcome. , $^{2,5-11,16,19,24}$ All patients in our series also underwent excision of the CCM and associated hematomas with an excellent overall clinical outcome.
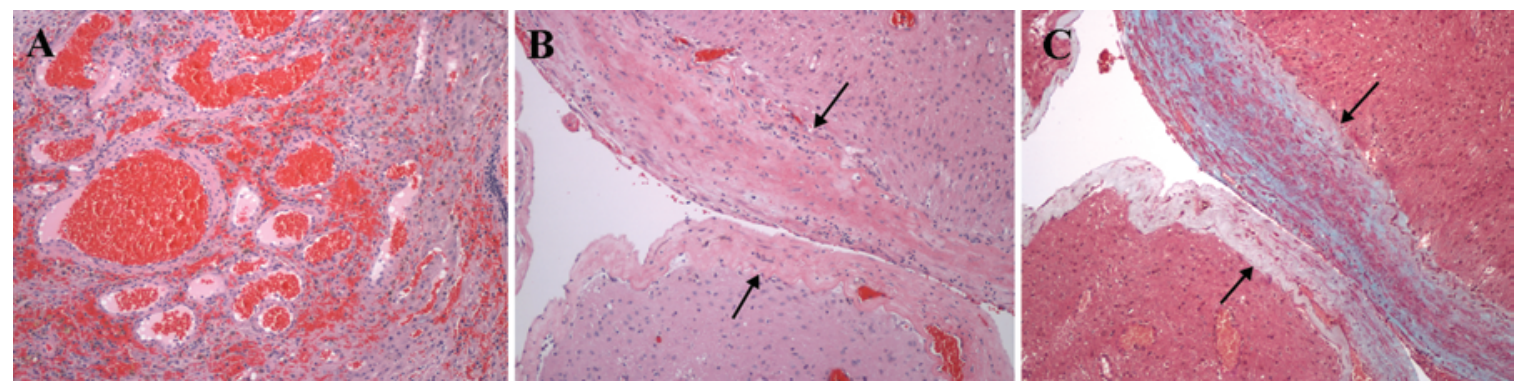

FIG. 6. Case 1. A: Numerous closely packed abnormal venous structures. B and C: Portions of an abnormally long and thick vessel wall (arrows) lacking black elastic fibers. This abnormal venous structure is directly opposed to cerebellar parenchyma (behind the arrows) and is suspected to represent the presumed DVA on neuroimaging. $H$ \& $E$ ( $A$ and $B)$, Movat's pentachrome stain $(C)$, original magnification $\times 100(A-C)$. Figure is available in color online only. 


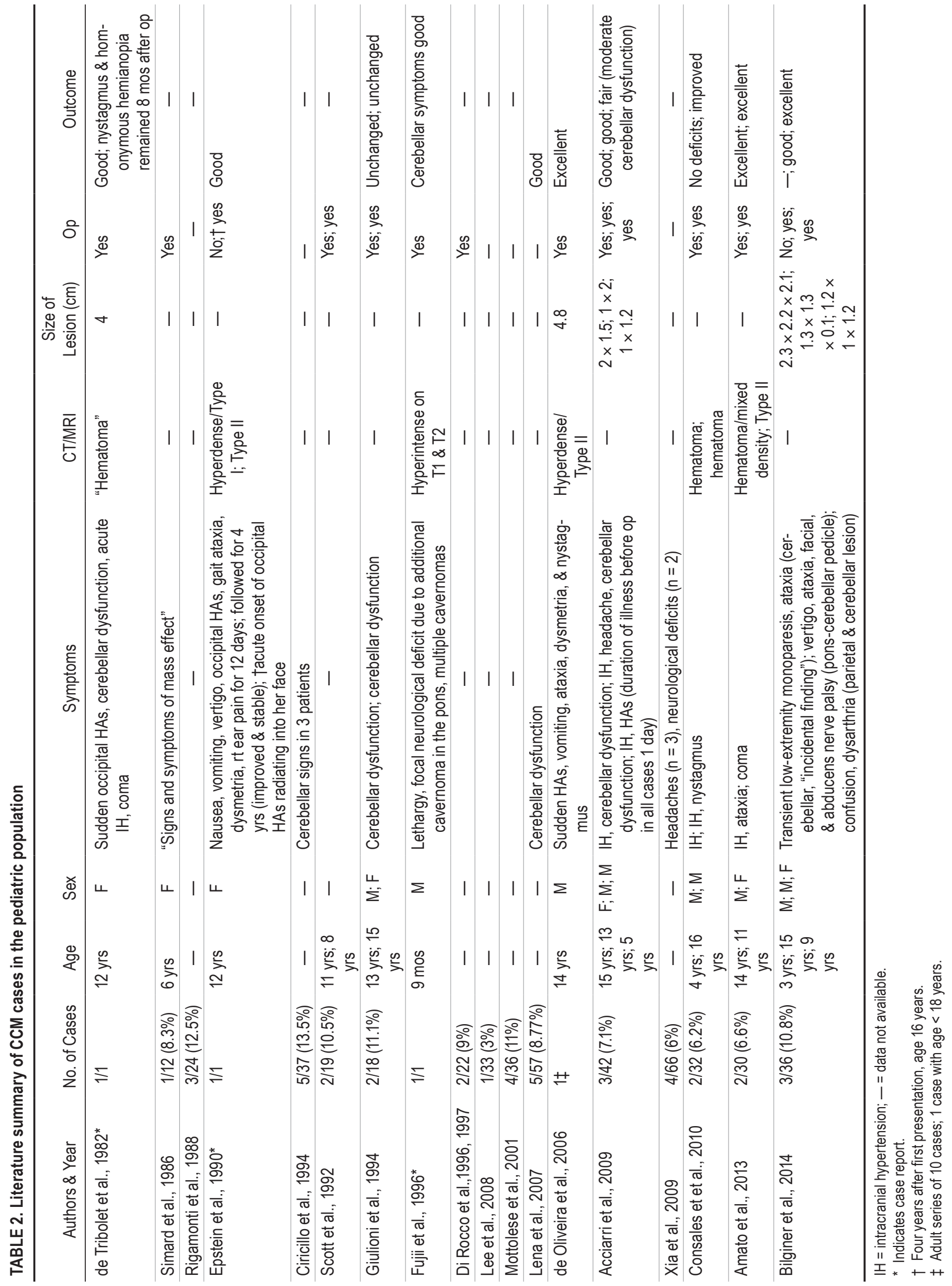


Considering their location, CCMs are straightforward to access surgically. However, they can become challenging to resect completely when parts of the lesion are scattered diffusely throughout adjacent brain parenchyma, as occurred in one of our patients.

\section{Conclusions}

Cerebellar CM presents acutely, in previously asymptomatic patients, with cerebellar parenchymal hemorrhage. The hematoma(s) are commonly large and can be mistaken for hemorrhage into a tumor. Cerebellar CM should be considered when the MRI characteristics suggest hemorrhage of varying age and the coexistence of a DVA. Surgery is a safe treatment option providing excellent clinical outcome in pediatric patients who are treated in a timely fashion. Subtotal resection should prompt ongoing clinical and imaging monitoring, as growth of residual CCM with repeat hemorrhage is possible.

\section{References}

1. Abdulrauf SI, Kaynar MY, Awad IA: A comparison of the clinical profile of cavernous malformations with and without associated venous malformations. Neurosurgery 44:41-47, 1999

2. Acciarri N, Galassi E, Giulioni M, Pozzati E, Grasso V, Palandri G, et al: Cavernous malformations of the central nervous system in the pediatric age group. Pediatr Neurosurg 45:81-104, 2009

3. Aiba T, Tanaka R, Koike T, Kameyama S, Takeda N, Komata T: Natural history of intracranial cavernous malformations. J Neurosurg 83:56-59, 1995

4. Al-Holou WN, O'Lynnger TM, Pandey AS, Gemmete JJ, Thompson BG, Muraszko KM, et al: Natural history and imaging prevalence of cavernous malformations in children and young adults. J Neurosurg Pediatr 9:198-205, 2012

5. Amato MC, Madureira JF, Oliveira RS: Intracranial cavernous malformation in children: a single-centered experience with 30 consecutive cases. Arq Neuropsiquiatr 71:220-228, 2013

6. Bigi S, Capone Mori A, Steinlin M, Remonda L, Landolt H, Boltshauser E: Cavernous malformations of the central nervous system in children: presentation, treatment and outcome of 20 cases. Eur J Paediatr Neurol 15:109-116, 2011

7. Bilginer B, Narin F, Hanalioglu S, Oguz KK, Soylemezoglu F, Akalan N: Cavernous malformations of the central nervous system (CNS) in children: clinico-radiological features and management outcomes of 36 cases. Childs Nerv Syst 30:1355-1366, 2014

8. Ciricillo SF, Cogen PH, Edwards MS: Pediatric cryptic vascular malformations: presentation, diagnosis and treatment. Pediatr Neurosurg 20:137-147, 1994

9. Consales A, Piatelli G, Ravegnani M, Pavanello M, Striano P, Zoli ML, et al: Treatment and outcome of children with cerebral cavernomas: a survey on 32 patients. Neurol Sci 31:117-123, 2010

10. de Oliveira JG, Rassi-Neto A, Ferraz FA, Braga FM: Neurosurgical management of cerebellar cavernous malformations. Neurosurg Focus 21(1):e11, 2006

11. de Tribolet N, Kaech D, Perentes E: Cerebellar haematoma due to a cavernous angioma in a child. Acta Neurochir (Wien) 60:37-43, 1982

12. Del Curling O Jr, Kelly DL Jr, Elster AD, Craven TE: An analysis of the natural history of cavernous angiomas. J Neurosurg 75:702-708, 1991

13. Di Rocco C, Iannelli A, Tamburrini G: Cavernomas of the central nervous system in children. A report of 22 cases. Acta Neurochir (Wien) 138:1267-1274, 1996

14. Di Rocco C, Iannelli A, Tamburrini G: Surgical management of paediatric cerebral cavernomas. J Neurosurg Sci 41:343347, 1997

15. Dillon WP: Cryptic vascular malformations: controversies in terminology, diagnosis, pathophysiology, and treatment. AJNR Am J Neuroradiol 18:1839-1846, 1997

16. Epstein MA, Berman PH, Schut L: Cavernous angioma presenting as atypical facial and head pain. J Child Neurol 5:27-30, 1990

17. Fortuna A, Ferrante L, Mastronardi L, Acqui M, d'Addetta R: Cerebral cavernous angioma in children. Childs Nerv Syst 5:201-207, 1989

18. Frim DM, Scott RM: Management of cavernous malformations in the pediatric population. Neurosurg Clin $\mathbf{N}$ Am 10:513-518, 1999

19. Fujii M, Arisawa M, Morimoto M, Kurisaka M, Mori K: Supra- and infratentorial multiple cavernous angiomas in an infant: report with MR evaluation of an unusual case. Childs Nerv Syst 12:564-567, 1996

20. Giulioni M, Acciarri N, Padovani R, Frank F, Galassi E, Gaist G: Surgical management of cavernous angiomas in children. Surg Neurol 42:194-199, 1994

21. Kim DS, Park YG, Choi JU, Chung SS, Lee KC: An analysis of the natural history of cavernous malformations. Surg Neurol 48:9-18, 1997

22. Kondziolka D, Lunsford LD, Kestle JR: The natural history of cerebral cavernous malformations. J Neurosurg 83:820824, 1995

23. Lee JW, Kim DS, Shim KW, Chang JH, Huh SK, Park YG, et al: Management of intracranial cavernous malformation in pediatric patients. Childs Nerv Syst 24:321-327, 2008

24. Lena G, Ternier J, Paz-Paredes A, Scavarda D: [Central nervous system cavernomas in children.] Neurochirurgie 53:223-237, 2007 (Fr)

25. Lim SC, Hong R, Kim YS, Jang SJ: Large cystic cavernous angioma of the cerebellum mimicking pilocytic astrocytoma. J Neurooncol 79:169-170, 2006

26. Mazza C, Scienza R, Beltramello A, Da Pian R: Cerebral cavernous malformations (cavernomas) in the pediatric agegroup. Childs Nerv Syst 7:139-146, 1991

27. Mottolese C, Hermier M, Stan H, Jouvet A, Saint-Pierre G, Froment JC, et al: Central nervous system cavernomas in the pediatric age group. Neurosurg Rev 24:55-73, 2001

28. Otten P, Pizzolato GP, Rilliet B, Berney J: [131 cases of cavernous angioma (cavernomas) of the CNS, discovered by retrospective analysis of 24,535 autopsies.] Neurochirurgie 35:82-83, 128-131, 1989 (Fr)

29. Papadias A, Taha A, Sgouros S, Walsh AR, Hockley AD: Incidence of vascular malformations in spontaneous intra-cerebral haemorrhage in children. Childs Nerv Syst 23:881-886, 2007

30. Perrini P, Lanzino G: The association of venous developmental anomalies and cavernous malformations: pathophysiological, diagnostic, and surgical considerations. Neurosurg Focus 21(1):e5, 2006

31. Porter RW, Detwiler PW, Spetzler RF, Lawton MT, Baskin JJ, Derksen PT, et al: Cavernous malformations of the brainstem: experience with 100 patients. J Neurosurg 90:50-58, 1999

32. Rigamonti D, Hadley MN, Drayer BP, Johnson PC, HoenigRigamonti K, Knight JT, et al: Cerebral cavernous malformations. Incidence and familial occurrence. N Engl J Med 319:343-347, 1988

33. Robinson JR, Awad IA, Little JR: Natural history of the cavernous angioma. J Neurosurg 75:709-714, 1991

34. Sarwar M, McCormick WF: Intracerebral venous angioma. Case report and review. Arch Neurol 35:323-325, 1978 
35. Scott RM, Barnes P, Kupsky W, Adelman LS: Cavernous angiomas of the central nervous system in children. J Neurosurg 76:38-46, 1992

36. Simard JM, Garcia-Bengochea F, Ballinger WE Jr, Mickle JP, Quisling RG: Cavernous angioma: a review of 126 collected and 12 new clinical cases. Neurosurgery 18:162-172, 1986

37. Willinsky R, Harper W, Wallace MC, Kucharczyk W, Montanera W, Mikulis D, et al: Follow-up MR of intracranial cavernomas. The relationship between haemorrhagic events and morphology. Interv Neuroradiol 2:127-135, 1996

38. Wurm G, Schnizer M, Nussbaumer K, Wies W, Holl K: Recurrent cryptic vascular malformation associated with a developmental venous anomaly. Br J Neurosurg 17:188-195, 2003

39. Xia C, Zhang R, Mao Y, Zhou L: Pediatric cavernous malformation in the central nervous system: report of 66 cases. Pediatr Neurosurg 45:105-113, 2009

40. Zhang P, Liu L, Cao Y, Wang S, Zhao J: Cerebellar cavernous malformations with and without associated developmental venous anomalies. BMC Neurol 13:134, 2013

\section{Author Contributions}

Conception and design: all authors. Acquisition of data: all authors. Analysis and interpretation of data: all authors. Drafting the article: all authors. Critically revising the article: all authors. Reviewed submitted version of manuscript: all authors. Approved the final version of the manuscript on behalf of all authors: Cochrane. Administrative/technical/material support: Cochrane, Knerlich-Lukoschus. Study supervision: Cochrane, KnerlichLukoschus.

\section{Correspondence}

David D. Cochrane, Division of Pediatric Neurosurgery, British Columbia Children's Hospital, 4480 Oak St., Rm. K3-216, Vancouver, BC V6H 3V4, Canada. email: dcochrane@cw.bc.ca. 